\title{
Force fields for divalent cations based on single-ion and ion-pair properties
}

\author{
Shavkat Mamatkulov, ${ }^{1}$ Maria Fyta, ${ }^{2,3}$ and Roland R. Netz ${ }^{1}$ \\ ${ }^{1}$ Fachbereich Physik, Freie Universität Berlin, 14195 Berlin, Germany \\ ${ }^{2}$ Physik Department, Technische Universität München, 85748 Garching, Germany \\ ${ }^{3}$ Institut für Computerphysik, Universität Stuttgart, 70569 Stuttgart, Germany
}

(Received 28 September 2012; accepted 6 December 2012; published online 10 January 2013)

\begin{abstract}
We develop force field parameters for the divalent cations $\mathrm{Mg}^{2+}, \mathrm{Ca}^{2+}, \mathrm{Sr}^{2+}$, and $\mathrm{Ba}^{2+}$ for molecular dynamics simulations with the simple point charge-extended (SPC/E) water model. We follow an approach introduced recently for the optimization of monovalent ions, based on the simultaneous optimization of single-ion and ion-pair properties. We consider the solvation free energy of the divalent cations as the relevant single-ion property. As a probe for ion-pair properties we compute the activity derivatives of the salt solutions. The optimization of the ionic force fields is done in two consecutive steps. First, the cation solvation free energy is determined as a function of the Lennard-Jones (LJ) parameters. The peak in the ion-water radial distribution function (RDF) is used as a check of the structural properties of the ions. Second, the activity derivatives of the electrolytes $\mathrm{MgY}_{2}, \mathrm{CaY}_{2}$, $\mathrm{BaY}_{2}, \mathrm{SrY}_{2}$ are determined through Kirkwood-Buff solution theory, where $\mathrm{Y}=\mathrm{Cl}^{-}, \mathrm{Br}^{-}, \mathrm{I}^{-}$. The activity derivatives are determined for the restricted set of $\mathrm{LJ}$ parameters which reproduce the exact solvation free energy of the divalent cations. The optimal ion parameters are those that match the experimental activity data and therefore simultaneously reproduce single-ion and ion-pair thermodynamic properties. For $\mathrm{Ca}^{2+}, \mathrm{Ba}^{2+}$, and $\mathrm{Sr}^{2+}$ such $\mathrm{LJ}$ parameters exist. On the other hand, for $\mathrm{Mg}^{2+}$ the experimental activity derivatives can only be reproduced if we generalize the combination rule for the anion-cation $\mathrm{LJ}$ interaction and rescale the effective cation-anion $\mathrm{LJ}$ radius, which is a modification that leaves the cation solvation free energy invariant. The divalent cation force fields are transferable within acceptable accuracy, meaning the same cation force field is valid for all halide ions $\mathrm{Cl}^{-}, \mathrm{Br}^{-}$, $\mathrm{I}^{-}$tested in this study. (@ 2013 American Institute of Physics. [http://dx.doi.org/10.1063/1.4772808]
\end{abstract}

\section{INTRODUCTION}

Polyvalent ions, especially divalent cations, play an essential role in physiological processes such as protein folding, modulation of enzyme activity, signal transduction, and many others. ${ }^{1-3}$ Due to their strong binding affinity to biomolecules, divalent cations promote fundamental changes in the functional activity of peptides. Most notably $\mathrm{Mg}^{2+}$ and $\mathrm{Ca}^{2+}$ are known to act very differently in the context of cellular physiology. ${ }^{4}$ As a specific example, $\mathrm{Ca}^{2+}$ is an important cofactor for blood clotting, meaning that blood plasma does not coagulate in the sole presence of $\mathrm{Mg}^{2+} .5,6$ To elucidate the influence of $\mathrm{Mg}^{2+}$ and $\mathrm{Ca}^{2+}$ on the blood clotting activity, the structural changes of the $\gamma$ - carboxyglutamic acid (GLA) domain, which is common to phylloquinone (vitamin K-dependent) proteins, ${ }^{6}$ were studied by X-ray diffraction at various ion concentrations. It has been observed that in the presence of $\mathrm{Mg}^{2+}$, active centers of the GLA domain, which are responsible for blood coagulations, retain disordered structure. However, if the molecules are surrounded by calcium ions, these active centers have a crystalline structure in which calcium is tightly bound. ${ }^{6}$ As another example, $\mathrm{X}$-ray crystallographic studies of the synthetic hexapeptide cyclo (-L-Pro-Gly-) $)_{3}$ show that in crystals containing $\mathrm{Ca}^{2+}$ ions, the cation is octahedrally surrounded by six carbonyl oxygens at an average distance of $2.26 \AA$. In a $\mathrm{Mg}^{2+}$ complex, the magnesium is octahedrally coordinated by three glycine carbonyl and three water oxygens at distances of 2.03 and
$2.11 \AA$, respectively. ${ }^{7}$ Also, $\mathrm{Mg}^{2+}$ and $\mathrm{Ca}^{2+}$ ions possess different abilities to induce adhesion and fusion of lipid vesicles or cells. ${ }^{8,9}$ Despite extensive ongoing research, many of the effects of divalent cations on the physiological activity of biomolecules are still not fully understood, partly because the fine differences between $\mathrm{Mg}^{2+}$ and $\mathrm{Ca}^{2+}$ are not accurately captured by standard simulation force fields.

From a computational perspective, ab initio quantum mechanical approaches are unbiased and therefore in principle ideal for the investigation of processes involving multivalent ions, ${ }^{10,11}$ but on the other hand are limited to relatively small systems due to the high computational demands. Classical atomistic simulations are less accurate but relatively inexpensive and thus allow to treat larger spatial and temporal scales. As a main drawback, classical simulations require accurate force fields (FF) in order to describe the microscopic interactions in various environments. Polarization effects were argued to improve the description of ion specificity. Despite many arguments in favor of polarizable force fields, the necessity of including polarizability for the accurate prediction of biomolecular properties, such as ligand-binding affinities, is debated. ${ }^{12}$ At the moment, non-polarizable force fields are still more widely used and quite successful in predicting binding affinities. ${ }^{13}$ The idea is that multi-body effects can be included effectively via the optimization of Lennard-Jones (LJ) parameters based on liquid state properties. It turns out that the effective ion size is crucial for accurately capturing ion 
TABLE I. Comparison of different divalent cation parameters. (1) AMBER FF (taken from the standard Amber data base), (2) CHARMM FF, ${ }^{17}$ (3) Åqvist $\mathrm{FF}^{18}{ }^{1}$ (4) Babu and Lim, ${ }^{19}$ and (5) this work. The sets $1,2,4$ were optimized using the TIP3P and set 3 using SPC water models. We also list the predicted solvation free energy and the first maximum of the RDF and compare with experimental numbers. All values were obtained using the SPC/E water model. The experimental solvation free energies are the sum of the divalent cation and the reference $\mathrm{Cl}^{-}$ion solvation free energies as compiled by Marcus. ${ }^{42}$

\begin{tabular}{|c|c|c|c|c|c|c|c|c|c|}
\hline Ion & Set & $\begin{array}{l}\sigma_{i O} \\
(\AA)\end{array}$ & $\begin{array}{c}\varepsilon_{i O} \\
(\mathrm{~kJ} / \mathrm{mol})\end{array}$ & $\begin{array}{l}\sigma_{i i} \\
(\AA)\end{array}$ & $\begin{array}{c}\varepsilon_{i i} \\
(\mathrm{~kJ} / \mathrm{mol})\end{array}$ & $\begin{array}{c}\Sigma_{\Delta G} \\
(\mathrm{~kJ} / \mathrm{mol})\end{array}$ & $\begin{array}{c}\Sigma_{\Delta G}^{\exp } \\
(\mathrm{kJ} / \mathrm{mol})\end{array}$ & $\begin{array}{l}\mathrm{R}_{1} \\
(\AA)\end{array}$ & $\begin{array}{l}\mathrm{R}^{\exp } \\
(\AA)\end{array}$ \\
\hline $\mathrm{Mg}^{2+}$ & 1 & 2.29 & 1.56 & 1.41 & 3.74 & -2513 & -2532 & 1.99 & 2.09 \\
\hline $\mathrm{Ca}^{2+}$ & 1 & 3.11 & 1.12 & 3.05 & 1.92 & -1970 & -2209 & 2.70 & 2.41 \\
\hline $\mathrm{Mg}^{2+}$ & 2 & 2.64 & 0.20 & 2.11 & 0.06 & -2540 & -2532 & 1.96 & 2.09 \\
\hline $\mathrm{Ca}^{2+}$ & 2 & 2.80 & 0.57 & 2.43 & 0.50 & -2254 & -2209 & 2.21 & 2.41 \\
\hline $\mathrm{Mg}^{2+}$ & 3 & 2.40 & 1.53 & 1.63 & 3.60 & -2424 & -2532 & 2.07 & 2.09 \\
\hline $\mathrm{Ca}^{2+}$ & 3 & 2.79 & 1.11 & 2.41 & 1.90 & -2168 & -2209 & 2.44 & 2.41 \\
\hline $\mathrm{Sr}^{2+}$ & 3 & 3.13 & 0.57 & 3.10 & 0.5 & -2037 & -2080 & 2.56 & 2.64 \\
\hline $\mathrm{Ba}^{2+}$ & 3 & 3.49 & 0.36 & 3.82 & 0.2 & -1892 & -1952 & 2.77 & 2.75 \\
\hline $\mathrm{Mg}^{2+}$ & 4 & 2.64 & 0.41 & 2.11 & 0.26 & -2450 & -2532 & 2.02 & 2.09 \\
\hline $\mathrm{Ca}^{2+}$ & 4 & 3.21 & 0.275 & 3.25 & 0.12 & -2081 & -2209 & 2.50 & 2.41 \\
\hline $\mathrm{Sr}^{2+}$ & 4 & 3.34 & 0.39 & 3.50 & 0.23 & -1962 & -2080 & 2.64 & 2.64 \\
\hline $\mathrm{Ba}^{2+}$ & 4 & 3.42 & 0.87 & 3.67 & 1.16 & -1810 & -1952 & 2.97 & 2.75 \\
\hline $\mathrm{Mg}^{2+}(1)$ & 5 & 2.40 & 0.62 & 1.63 & 0.59 & -2532 & -2532 & 1.96 & 2.09 \\
\hline $\mathrm{Mg}^{2+}(2)$ & 5 & 2.90 & 0.05 & 2.63 & 0.004 & -2531 & -2532 & 1.98 & 2.09 \\
\hline $\mathrm{Ca}^{2+}$ & 5 & 2.79 & 0.78 & 2.41 & 0.94 & -2209 & -2209 & 2.38 & 2.41 \\
\hline $\mathrm{Sr}^{2+}$ & 5 & 3.13 & 0.40 & 3.10 & 0.25 & -2080 & -2080 & 2.51 & 2.64 \\
\hline $\mathrm{Ba}^{2+}$ & 5 & 3.50 & 0.22 & 3.82 & 0.074 & -1952 & -1952 & 2.69 & 2.75 \\
\hline
\end{tabular}

specific effects and must be correctly represented by the 6$12 \mathrm{LJ}$ potential, which is commonly used for the modeling of ions, together with combination (or mixing) rules to describe interactions between different atoms or ions.

The force field parameters reported in literature for divalent cations are typically optimized based on single-ion properties in solution or with respect to experimental data in the crystalline state. In practice, these force fields often fail to reproduce electrolyte thermodynamic properties or ion specific effects at finite concentrations even for simple ionic solutions. ${ }^{14-16}$ Therefore recently much attention has been given on the development of force fields that can reproduce several thermodynamic properties of ionic solutions simultaneously at finite concentrations. A number of non-polarizable models for divalent cations are available in the literature. ${ }^{17-20}$ Table I shows the ion parameters of a few widely used force fields for divalent cations. All these force fields were optimized to reproduce a variety of thermodynamic properties including solvation free energy, the first peak of the ionwater radial distribution function, crystal lattice parameters, etc. However, their development was based mostly on singleion properties and thus these force fields are a priori reliable only in simulations at low salt concentrations. As an example, commonly available force fields as implemented in CHARMM, GROMOS, and AMBER for divalent cations have failed to describe ion specific effects in ion channels. Molecular dynamics (MD) studies of binding and permeability of monovalent and divalent cations such as $\mathrm{Ca}^{2+}, \mathrm{Mn}^{2+}$, $\mathrm{Ba}^{2+}$ in a gramicidin A channel showed that non-polarizable force fields can describe the binding configurations of monovalent ions within the gramicidin A channel but cannot describe energetics of binding and blocking of the divalent cations. ${ }^{21,22}$ It is clear from Table I that parameters for one and the same ion significantly differ in different parameter sets. This noticeable difference cannot be simply explained by the fact that these were developed in combination with different water models, as the influence of the water models is relatively small. ${ }^{23}$ It is rather based on different optimization strategies and partial arbitrariness which is inherent in the force field parameterization, specifically when only single-ion properties are used as probes for the optimization of two force field parameters.

In this work, our aim is to develop force field parameters for the divalent cations $\mathrm{Mg}^{2+}, \mathrm{Ca}^{2+}, \mathrm{Ba}^{2+}$, and $\mathrm{Sr}^{2+}$ in conjunction with the simple point charge-extended (SPC/E) water model, as relevant for classical simulations of biological macromolecular processes. The optimization is performed in two steps: First we determine the solvation free energy of divalent cations in the entire parameter space of Lennard-Jones parameters, $\sigma$ and $\varepsilon$. As a consistency check of the resulting force fields we also determine the effective radii of the ions from the first peak in the ion-water radial distribution function. Determining the combinations of the ion-water LJ diameter $\sigma_{i O}$ and the ion-water LJ strength $\varepsilon_{i O}$, which reproduce the experimental solvation free energy, defines a curve in the parameter plane spanned by $\sigma_{i O}$ and $\varepsilon_{i O}$. We then compute the activity derivatives of the electrolytes such as $\mathrm{MgY}_{2}$, $\mathrm{CaY}_{2}, \mathrm{BaY}_{2}, \mathrm{SrY}_{2}$, where $\mathrm{Y}=\mathrm{Cl}^{-}, \mathrm{Br}^{-}, \mathrm{I}^{-}$on the line on which the experimental solvation free energy is reproduced. While the ion solvation free energy describes the binding of water to the ion in the solvation shell, the activity derivatives are a sensitive measure of the ion-ion pairing properties. ${ }^{24,25}$ In the end, the optimal ion parameter corresponds to the $\mathrm{LJ}$ pair combination that gives a value of the activity derivative 
which most closely matches the respective experimental values. We show that for $\mathrm{Ca}^{2+}, \mathrm{Ba}^{2+}$, and $\mathrm{Sr}^{2+}$ at least one $\mathrm{LJ}$ pair combination exists that simultaneously matches experimental solvation and activity data. This is not the case for $\mathrm{Mg}^{2+}$ ions. Here we introduce a scaling factor in the cationanion $\mathrm{LJ}$ interaction that quantifies deviations from the standard combination rule for the effective LJ diameter and allows to reproduce the activity coefficient without modifying the single $\mathrm{Mg}^{2+}$ ion solvation free energy (which was optimized in the first step). By basing our optimization on both single-ion and ion-pair properties, we substantially improve the existing optimization schemes.

This paper is organized as following: In Sec. II, we present the methodology used in this study, in Sec. III we discuss the results for the calculated salt activity derivatives and force field parameters generated for divalent cations. In Sec. IV, we briefly conclude and discuss possible future work.

\section{METHODOLOGY}

\section{A. Molecular dynamics (MD) simulations}

The pair interaction potentials between particles $U_{i j}$ are modeled through a sum of the Coulomb interaction and a $\mathrm{LJ}$ term:

$$
U_{i j}\left(r_{i j}\right)=\frac{q_{i} q_{j}}{r_{i j}}+\left[\frac{A_{i j}}{r_{i j}^{12}}-\frac{B_{i j}}{r_{i j}^{6}}\right],
$$

where $q_{i}, q_{j}$ are the charges of the atoms $i, j$ and $r_{i j}$ is the distance between these atoms. Via $\mathrm{A}_{i j}=4 \varepsilon_{i j} \sigma_{i j}^{12}$ and $\mathrm{B}_{i j}$ $=4 \varepsilon_{i j} \sigma_{i j}^{6}$, the LJ effective radius and interaction strength $\sigma_{i j}$ and $\varepsilon_{i j}$ are defined. We use the Lorentz-Berthelot combination rules for the Lennard-Jones parameters:

$$
\varepsilon_{i j}=\sqrt{\varepsilon_{i} \varepsilon_{j}} ; \quad \sigma_{i j}=\frac{\sigma_{i}+\sigma_{j}}{2},
$$

where $i, j$ correspond to the index of the atoms and ions, but also consider deviations from these standard combination rules, as will be discussed later on. Note that the NBFIX modification in the CHARMM MD simulation software ${ }^{26}$ also allows to consider alternative mixing rules by specifying individual atom-atom van der Waals interactions. We employ the SPC/E water model, ${ }^{27}$ which assigns partial charges of -0.8476 and 0.4238 to oxygen and hydrogen, respectively. The geometry is fixed at a bond length of $1.0 \AA$ and a bond angle of $109.47^{\circ}$ using the SHAKE algorithm. ${ }^{28}$

The single-ion solvation properties in water are studied employing the thermodynamic integration (TI) ${ }^{29}$ scheme implemented in AMBER 8.0 package. ${ }^{20}$ For the structural analysis and the calculation of activity coefficients of electrolyte aqueous solutions we use the GROMACS 4.5.4 software. ${ }^{30,31}$ For the single-ion solvation properties, a single ion is placed in a cubic box of size $\mathrm{L}=25 \AA \AA$ containing 506 SPC/E water molecules. A cut-off distance of $r_{c}=9.0 \AA$ is chosen for the Lennard-Jones interactions and long range corrections for energy and pressure due to dispersion interactions is used in all simulations. Periodic boundary conditions are applied in all three directions, and a particle-mesh Ewald summation (PME) with a grid spacing of $1 \AA$ in conjunction with tinfoil boundary conditions is used to handle long-ranged electrostatic forces. ${ }^{32}$ The free energy of solvation is computed from molecular dynamics simulations performed with a time step of $1 \mathrm{fs}$. The simulations are carried out in the NPT ensemble using the Berendsen weak coupling method to keep the temperature at $300 \mathrm{~K}$ and the reference pressure at $1 \mathrm{bar}^{33}$

The radial distribution functions of the salt solutions are obtained using GROMACS, from atomistic simulations performed also in the NPT ensemble, at a pressure of $1 \mathrm{bar}$ and temperature $\mathrm{T}=300 \mathrm{~K}$. The initial configurations of the electrolyte solutions are generated from a cubic box $(\mathrm{L}=40 \AA)$ of 2180 equilibrated SPC/E water molecules by randomly replacing water by ions until the required concentration is attained. After the replacements, there are 19 cations and 38 anions in the box, corresponding to a molality (mol $/ \mathrm{kg}$ ) of about $0.5 \mathrm{~m}$. A few simulations are performed for larger systems to check for finite-size effects. Here we take a larger box with a size of $\mathrm{L}=60 \AA$ A containing 6966 water molecules, 65 $\mathrm{Mg}^{2+}$, and $130 \mathrm{Cl}^{-}$ions. The particle mesh Ewald sum technique is used to evaluate electrostatic interactions using cubic interpolations and a grid spacing of $1.2 \AA$ for the reciprocal space sum, coupled with tinfoil boundary conditions. Simulations are performed with a 2 fs time step. For gathering better statistics the trajectory of particles is written after every $0.2 \mathrm{ps}$ and total simulation time is $150 \mathrm{~ns}$. All parameters used in the MD simulations have been previously checked to lead to consistent results for the systems under study. ${ }^{24,34}$

\section{B. Free energy calculation and correction terms}

The solvation free energies of the divalent cations are calculated using thermodynamic integration (TI) ${ }^{29}$ and the identity:

$$
\Delta G_{s i m}=\int_{0}^{1}\left\langle\frac{\partial H_{\lambda}\left(\lambda_{L J}\right)}{\partial \lambda_{L J}}\right\rangle d \lambda_{L J}+\int_{0}^{1}\left\langle\frac{\partial H_{\lambda}\left(\lambda_{C}\right)}{\partial \lambda_{C}}\right\rangle d \lambda_{C},
$$

where $H_{\lambda}$ is the Hamiltonian of the system, $\lambda_{L J}$ and $\lambda_{C}$ are the $\mathrm{LJ}$ and charge transition coordinates which are 0 in the initial state and 1 in the final state. To compute the solvation free energy of the divalent cations we use the simulation data of monovalent cations obtained in our previous work. ${ }^{35}$ The solvation path is split in three separate processes: first, a neutral van der Waals particle is created, then assigned a charge of 1 in a second step (simulation data from $^{35}$ ), and this monovalent cation charge is increased to $\mathrm{q}=2$ in the last step. Along the transition path, the $\lambda$-dependent Hamiltonian is defined as

$$
\begin{gathered}
H_{\lambda}\left(\lambda_{L J}, \lambda_{C}\right)=H\left\{q_{\text {ion }}=\lambda_{C} q, \quad \sigma_{\text {ion }}=\left[1-\left(1-\lambda_{L J}\right)^{k}\right] \sigma,\right. \\
\left.\varepsilon_{\text {ion }}=\left[1-\left(1-\lambda_{L J}\right)^{k}\right] \varepsilon\right\} .
\end{gathered}
$$

We set the exponent $k$ in this equation equal to $k=6$ to avoid divergences in $\partial H_{\lambda}\left(\lambda_{L J}\right) / \partial \lambda_{L J}$. Integrations are performed through a 12-point Gaussian quadrature with $\lambda$ $\in\{0.00922,0.04794,0.11505,0.20634,0.31608,0.43738$, $0.56262,0.68392,0.79366,0.88495,0.95206,0.99078\}$. For every value of $\lambda$ we perform a 250 ps simulation of which the first $50 \mathrm{ps}$ are discarded for equilibration.

The raw ionic solvation free energy computed in the simulations is sensitive to the simulation scheme (system shape, 
periodic or finite system) and treatment of the electrostatic forces (Ewald sum, cut-off based, etc). Therefore for comparison with experimental data, several corrections have to be applied to the raw simulation data. The correction term accounting for finite size effects ${ }^{36}$ related to the system size and the ion size for an ion with charge of $z e$, where $e$ is the unit charge and $z$ its valency, reads

$$
\Delta G_{f s}=\frac{z^{2} N_{A} e^{2}}{4 \pi \varepsilon_{0}^{2}}\left[-\frac{\xi_{e w}}{2 \varepsilon_{r}}+\left(1+\frac{1}{\varepsilon_{r}}\right)\left(\frac{2 \pi R_{1}^{2}}{3 L^{3}}-\frac{4 \pi^{2} R_{1}^{5}}{45 L^{6}}\right)\right],
$$

where $N_{A}$ is Avogadro's number, $R_{1}$ is the effective radius of the ion, estimated as the first peak in the ion-oxygen radial distribution function, and $\varepsilon_{r}=71$ is the relative dielectric constant of the SPC/E water. ${ }^{37}$ The Wigner potential is $\xi_{\text {ew }}$ $=-2.837279 / \mathrm{L}$, where $\mathrm{L}$ is the simulation box size in nm. ${ }^{38,39}$

Experimental values of the solvation free energies are usually given with respect to a hypothetical transfer of ions from the ideal gas phase of $p_{0}=1$ atm pressure to the ideal solution under pressure of $p_{1}=24.6 \mathrm{~atm}$, corresponding to the density of $1 \mathrm{~mol} / \mathrm{l}$. Thus, it is also necessary to include a correction term related to the compression of the gas:

$$
\Delta G_{\text {press }}=N_{A} k_{B} T \ln \left(p_{1} / p_{0}\right)=7.9 \mathrm{~kJ} / \mathrm{mol},
$$

where $k_{B} T$ is the thermal energy.

In the experiments, the ions have to pass the air-water interface in order to enter into the aqueous phase. Whenever single-ion solvation free energies are reported, the effect related to the electrostatic potential jump at the air-water interface must be included. Consequently, the experimental values of solvation free energy for single ions depend sensitively on the rather ill-characterized surface potential properties. In this paper we eliminate this problem by fitting only neutral ionpair properties. Nevertheless, in order to present physically meaningful single-ion solvation free energies, we choose the surface potential as $\phi_{\text {surf }}=-0.527 \mathrm{~V} .{ }^{40}$ The corresponding free energy correction term is

$$
\Delta G_{\text {surf }}=N_{A} z \times e \phi_{\text {surf }}=-z \times 50.8 \mathrm{~kJ} / \mathrm{mol} .
$$

Hence, the total single-ion solvation free energy is given by

$$
\Delta G_{\text {solv }}=\Delta G_{\text {sim }}+\Delta G_{f s}+\Delta G_{\text {surf }}+\Delta G_{\text {press }} .
$$

\section{Parameter space sampling and fitting}

We sample the LJ parameters $\sigma_{i j}$ and $\varepsilon_{i j}$ on a $7 \times 9$ grid. For divalent cations the range $\sigma_{i j}=2.2-4.25 \AA$ and $\varepsilon_{i j}$ $=0.02-1.28 \mathrm{~kJ} / \mathrm{mol}$ is studied. For an efficient optimization of $\sigma_{i j}$ and $\varepsilon_{i j}$, the surfaces of the solvation free energy and the effective ion radius are fitted by polynomial expressions as

$$
\begin{gathered}
\Delta G_{\text {solv }}=\sum_{i=0}^{3} \sum_{j=0}^{3} g_{i j} \sigma^{i} \varepsilon^{j / 2}, \\
R_{1}=\sum_{i=0}^{3} \sum_{j=0}^{3} \rho_{i j} \sigma^{i} \varepsilon^{j / 2} .
\end{gathered}
$$

The coefficients $g_{i j}$ and $\rho_{i j}$ are determined through the minimization of the root-mean-square (rms) error, yielding
TABLE II. Ion-water and ion-ion interaction parameters used in the MD simulations for the anions.

\begin{tabular}{lccccc}
\hline \hline Ion & $\sigma_{i O}(\AA)$ & $\varepsilon_{i O}(\mathrm{~kJ} / \mathrm{mol})$ & $\sigma_{i i}(\AA)$ & $\varepsilon_{i i}(\mathrm{~kJ} / \mathrm{mol})$ & Ref. \\
\hline $\mathrm{Cl}^{-}$ & 3.78 & 0.52 & 4.40 & 0.41 & 43 \\
$\mathrm{Br}^{-}$ & 3.90 & 0.52 & 4.63 & 0.41 & 43 \\
$\mathrm{I}^{-}$ & 4.25 & 0.32 & 5.33 & 0.16 & 35 \\
\hline \hline
\end{tabular}

rms errors of the fitting surfaces for $\Delta G_{s o l v}$ and $\mathrm{R}_{1}$ (the effective radius of ions) of $\pm 1.5 \mathrm{~kJ} / \mathrm{mol}$ and $0.009 \AA$, respectively.

\section{Fitting to ion-pair data}

Extracting single-ion solvation free energies from experimental data usually relies on the solvation free energy of the proton $\Delta G_{\text {solv }}\left(H^{+}\right)$. However, the proton solvation free energy is perturbed by the surface potential of water, which is not exactly known and prone to errors. A popular estimate for $\Delta G_{\text {solv }}\left(H^{+}\right)$is that of Tissandier et al. ${ }^{41}$ $(-1104.5 \mathrm{~kJ} / \mathrm{mol})$. This value is $50 \mathrm{~kJ} / \mathrm{mol}$ lower than the other commonly used value of $\operatorname{Marcus}^{42}(-1056 \mathrm{~kJ} / \mathrm{mol})$. Experimental solvation free energy data are more robust for neutral ion-pairs, for which the water surface potential drops out. In order to optimize ion-pair properties, the definition of the LJ parameters for a reference ion is required. We choose the chloride ion with the commonly used Smith-Dang parameters $^{43}$ given in Table II. Taking Smith-Dang parameters for $\mathrm{Cl}^{-} \sigma_{i O}=3.78 \AA$ and $\varepsilon_{i O}=0.52 \mathrm{~kJ} / \mathrm{mol}$ and using the data from Ref. 35 , the solvation free energy value of $\Delta G_{\text {solv }}=-306 \mathrm{~kJ} / \mathrm{mol}$ is obtained, which is close to the absolute solvation free energy of $\mathrm{Cl}^{-}$based on Tissandier's estimate for the absolute proton solvation energy, given by $\Delta G_{\text {solv }}=-304.2 \mathrm{~kJ} / \mathrm{mol}^{41}{ }^{4}$ For the divalent cations in this study, the solvation free energies to be optimized are always the sum of the cationic and the chloride free energy, respectively,

$$
\Sigma_{\Delta G}=\Delta G_{s o l v}+z \times \Delta G_{s o l v}\left(C l^{-}\right),
$$

where $z=2$ is the valency of the cation.

\section{E. Kirkwood-Buff theory}

The Kirkwood-Buff (KB) solution theory describes the relationship between pair correlation functions and thermodynamic system properties and is a powerful tool for the study of complex fluids as well as salt solutions. We will use KB theory to obtain the activity derivatives of the salt solutions, as was done before in similar studies. ${ }^{44}$ The KB theory connects integrals over pair distribution functions, the so-called $\mathrm{KB}$ integrals, to thermodynamic quantities such as isothermal compressibilities, partial molar volumes, and in particular solution activity derivatives.

The KB integrals are defined as ${ }^{15}$

$$
G_{i j}=4 \pi \int_{0}^{\infty}\left(g_{i j}-1\right) r^{2} d r
$$

where $g_{i j}$ is the radial distribution function between species $i$ and $j$. Using these $\mathrm{KB}$ integrals, we can compute the 
derivative of the activity coefficients, $a_{c c}$, of electrolyte solutions

$$
a_{c c}=1+\left(\frac{\partial \ln y_{c}}{\partial \ln \rho_{c}}\right)_{\left.\right|_{p, T}}=\frac{1}{1+\rho_{c}\left(G_{c c}-G_{c w}\right)},
$$

where the molar activity coefficient $y_{c}$ is defined via $a_{c}=\rho_{c} y_{c}$, while $\rho_{c}$ is the cosolvent number density and $a_{c}$ is the activity.

The following integrals are defined: ${ }^{44}$

$$
\begin{gathered}
G_{c c}=\left(\frac{n_{+}}{n}\right)^{2} G_{++}+\left(\frac{n_{-}}{n}\right)^{2} G_{--}+\left(\frac{n_{+} n_{-}}{n^{2}}\right)\left(G_{+-}+G_{-+}\right) \\
G_{c w}=G_{w c}=\left(\frac{n_{+}}{n}\right) G_{+w}+\left(\frac{n_{-}}{n}\right) G_{-w}
\end{gathered}
$$

where $n=n_{+}+n_{-}$is the total number density of ions in the solution, while $n_{+}, n_{-}$are the number densities of cations and anions in the solution.

For monovalent ions these equations take the form, ${ }^{25}$

$$
G_{c c}^{(1)}=\frac{1}{4}\left[G_{++}+G_{--}+2 G_{+-}\right]
$$

and

$$
G_{c w}^{(1)}=G_{w c}^{(1)}=\frac{1}{2}\left(G_{+w}+G_{-w}\right)
$$

as given in Refs. 24 and 44 . (Note, that the factor $\frac{1}{2}$ in the second equation is missing in Ref. 24 due to a typing error.)

For divalent salt the number densities can be written as

$$
n_{+}=\frac{1}{3} n ; \quad n_{-}=\frac{2}{3} n
$$

and the expressions for the divalent ions are now revised to

$$
\begin{gathered}
G_{c c}^{(2)}=\frac{1}{9}\left[G_{++}+4\left(G_{--}+G_{+-}\right)\right], \\
G_{c w}^{(2)}=G_{w c}^{(2)}=\frac{1}{3} G_{+w}+\frac{2}{3} G_{-w} .
\end{gathered}
$$

Additional details on the KB theory can be found elsewhere. ${ }^{15,44,45}$

\section{F. Finite size corrections}

The structural properties of salt solutions are described through the radial distribution functions (RDFs) $g_{i j}$, which describe how the atomic density varies as a function of the distance $r$ from a particular atom. The RDFs are directly obtained from the MD simulations trajectories. In order to achieve accurate results, finite size effects need to be accounted for. Of particular importance is the condition that the RDFs should reach unity at large distances ${ }^{46}$ Here we introduce a correcting factor, so that the RDF used in the calculations of the Kirkwood-Buff integrals (KBIs) is written as $g_{i j}\left(r^{\prime} \rho\right)=f(\rho) g_{i j}^{s i m}(r)$ with a prefactor $f(\rho)$ of the order of unity adjusted such as to ensure correct asymptotic behavior at large distances.

We have explicitly checked box size and relaxation effects on the activity coefficient derivative values obtained through Kirkwood-Buff theory. We have ana-
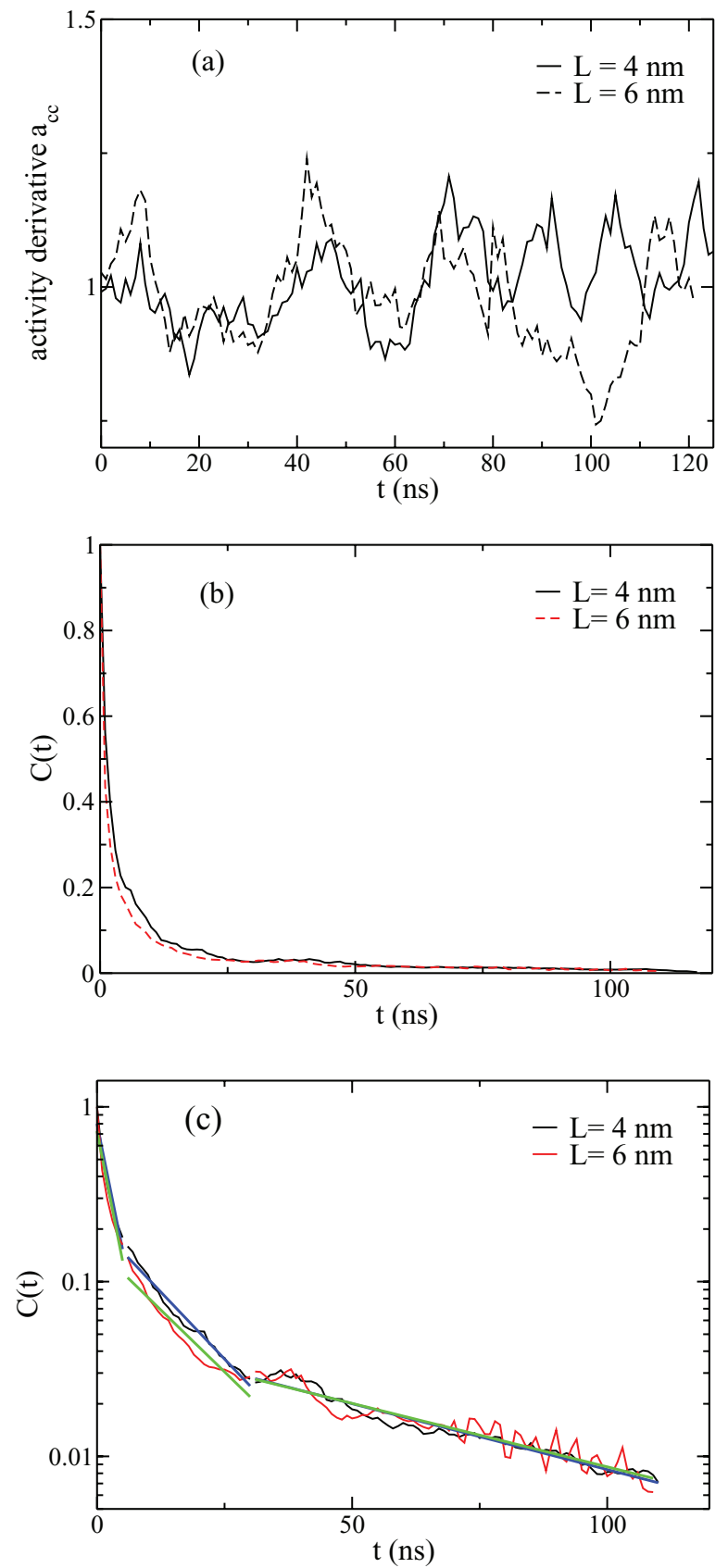

FIG. 1. (a) Activity derivative of a $0.5 \mathrm{~m} \mathrm{MgCl} 2$ solution as a function of simulation time for different simulation box sizes $L=40 \AA$ and $L=60 \AA$, containing 6426 and 21043 atoms, respectively. The mean activity derivative is $\mathrm{a}_{c c}(\mathrm{~L}=40 \AA)=0.985 \pm 0.03$ and $\mathrm{a}_{c c}(\mathrm{~L}=60 \AA)=0.978 \pm 0.03$, respectively. (b) The autocorrelation function of the activity derivative is shown to decay over about $10 \mathrm{ps}$ for both box sizes. (c) The autocorrelation function in a logarithmic plot, straight lines represent exponential fit functions.

lyzed the $\mathrm{MD}$ results for, $\mathrm{a}_{c c}$, of $\mathrm{MgCl}_{2}$ at electrolyte density of $0.5 \mathrm{~m}$ in two boxes with different sizes $\mathrm{L}=40$ and $60 \AA$ and have compared its variation with time and its autocorrelation function. Figure 1(a) shows $\mathrm{a}_{c c}$ for $\mathrm{MgCl}_{2}$ as a function of time for the two different system sizes. The $\mathrm{a}_{c c}$ values were averaged in time frames of $10 \mathrm{~ns}$. Despite the large fluctuations, the mean value of the activity derivatives are similar for both boxes within the error of the calculation. The average activity derivative for $\mathrm{MgCl}_{2}$, obtained from a simulation of total duration $150 \mathrm{~ns}$, is $\mathrm{a}_{c c}(\mathrm{~L}=40 \AA$ ) 
$=0.985 \pm 0.03$ and $\mathrm{a}_{c c}(\mathrm{~L}=60 \AA)=0.978 \pm 0.03$ for the two different boxes. The autocorrelation functions of the activity derivatives are shown in Figs. 1(b) and 1(c) for both simulation boxes. In general, the autocorrelation function describes the dynamics of an observable and is defined as

$$
C(t)=\frac{1}{T} \int_{0}^{T} d t^{\prime} \frac{\left\langle a_{c c}\left(t^{\prime}\right) a_{c c}\left(t^{\prime}+t\right)\right\rangle}{\left\langle a_{c c}(0) a_{c c}(0)\right\rangle},
$$

where $T$ is the time over which the averaging is performed. The mean-relaxation time is obtained by fitting exponentials in separate time ranges, as shown in Fig. 1(c). By doing so we obtain three distinct relaxation times, given by $\tau_{1} \approx 3 \mathrm{~ns}$, $\tau_{2} \approx 15 \mathrm{~ns}$, and $\tau_{3} \approx 50 \mathrm{~ns}$. The occurrence of three relaxation times is presumably related to distinct water-water, water-ion and ion-ion relaxation phenomena. The main point is that the dominant relaxation times are smaller than the total simulation time.

\section{RESULTS AND DISCUSSION}

\section{A. Solvation free energy and radial distribution function}

The simulation results for the solvation free energy of divalent cations and the corresponding correction terms, as well as the polynomial fit parameters for the solvation free energy and the first peak in the ion-water radial distribution function are given in Tables 1 and 2 in the supplementary material. ${ }^{55}$ The solvation of divalent cations is a very favorable process and the corresponding free energies range from $-2240 \mathrm{~kJ} / \mathrm{mol}$ for $\sigma_{i O}=2.2 \AA$ and $\varepsilon_{i O}=0.02 \mathrm{~kJ} / \mathrm{mol}$ to $-859 \mathrm{~kJ} / \mathrm{mol}$ for $\sigma_{i O}$ $=4.256 \AA$ and $\varepsilon_{i O}=1.277 \mathrm{~kJ} / \mathrm{mol}$. Uncertainty in the solvation free energy and radius is estimated by statistical error propagation. Figure 2 shows the simulation results for $\Delta G_{\text {solv }}$
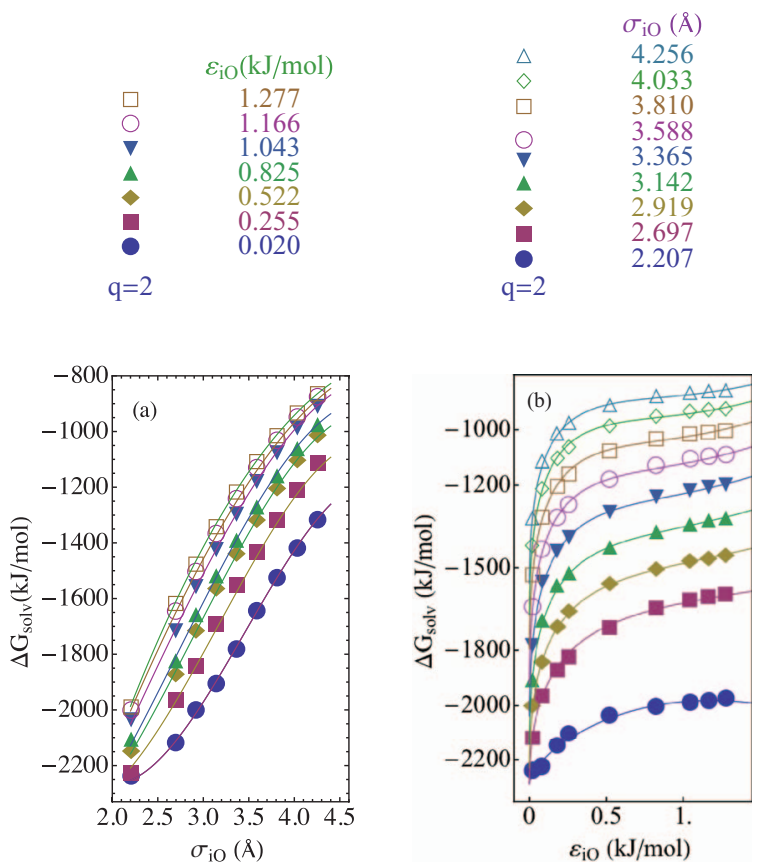

FIG. 2. Single-ion solvation free energy $\Delta G_{\text {solv }}$ as a function of the Lennard-Jones parameters, $\sigma_{i O}$ and $\varepsilon_{i O}$. The symbols show the points obtained using thermodynamic integration; the lines represent cuts of the fitted free energy surface according to Eq. (9)
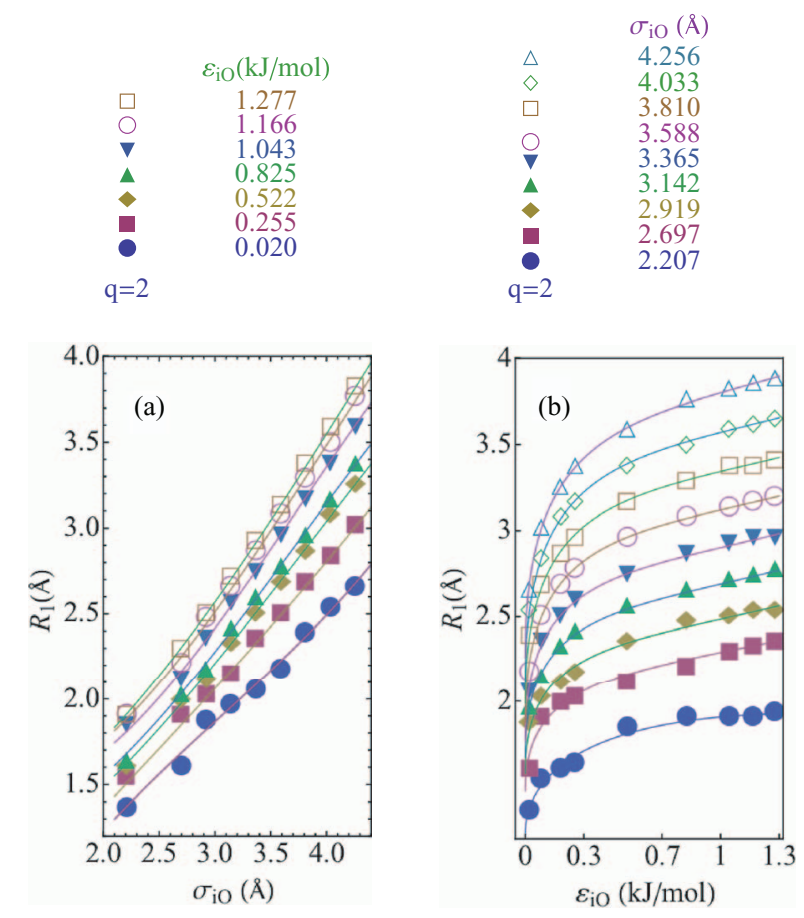

FIG. 3. Position of the first maximum in the divalent cation-water RDF $\left(\mathrm{R}_{1}\right)$ as a variation of the Lennard-Jones parameters of $\sigma_{i O}$ and $\varepsilon_{i O}$. The symbols show the MD results; the lines represent the cuts of the fitted surface according to Eq. (10).

as a function of $\sigma_{i O}$ and $\varepsilon_{i O}$ for all grid points. The lines represent cuts of the free energy surface obtained using the polynomial function Eq. (9). With increasing $\sigma_{i O}$ and $\varepsilon_{i O}$ the solvation process becomes less favorable and the solvation free energy increases, as seen in Fig. 2.

The ion-water radial distribution function is calculated for every combination of $\sigma_{i O}$ and $\varepsilon_{i O}$ from $4 \mathrm{~ns}$ simulations. The first maximum in the ion-water RDF, denoted as $\mathrm{R}_{1}$, is a measure of the solvation radius. The simulation results for $\mathrm{R}_{1}$ as a function of $\sigma_{i O}$ and $\varepsilon_{i O}$ are shown in Fig. 3, which again monotonically increase with the LJ parameters $\sigma_{i O}$ and $\varepsilon_{i O}$ similarly to the free energy of solvation. The symbols correspond to the simulation data, while the lines denote cuts of the fitted $\mathrm{R}_{1}$ surface at constant $\sigma_{i O}$ and $\varepsilon_{i O}$, obtained through Eq. (10).

We show the calculated $\Sigma_{\Delta G}$ and $\mathrm{R}_{1}$ isolines based on experimental data for $\mathrm{Mg}^{2+}, \mathrm{Ca}^{2+}, \mathrm{Sr}^{2+}, \mathrm{Ba}^{2+}$ in Fig. 4. In this figure other commonly used force fields found in the literature are plotted for comparison. Set (1) represents the AMBER force field from the standard database. The CHARMM force field (2) for $\mathrm{Mg}^{2+}$ is optimized to reproduce the solvation free energy based on Tissandier's compilation and indeed lies perfectly on the solid line defining the experimental solvation free energy. The Åqvist force field (3) perfectly matches the experimental values of the first peak in the ion water radial distribution function, but underestimates solvation free energies. Babu and $\operatorname{Lim}^{19}$ developed a divalent cation force field (4) based on relative solvation free energies and first shell coordination numbers of ions. From Fig. 4 it is evident that some of these force fields found in the literature can reproduce the experimental values for $\Sigma_{\Delta G}$, others 


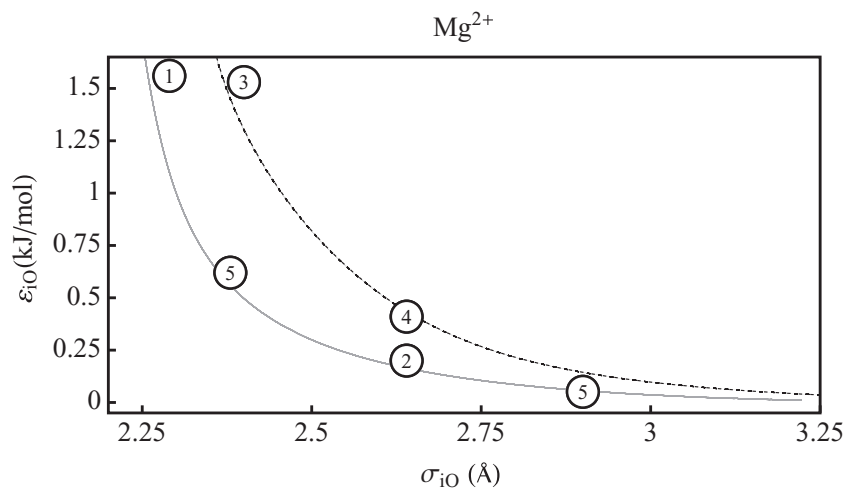

(a)

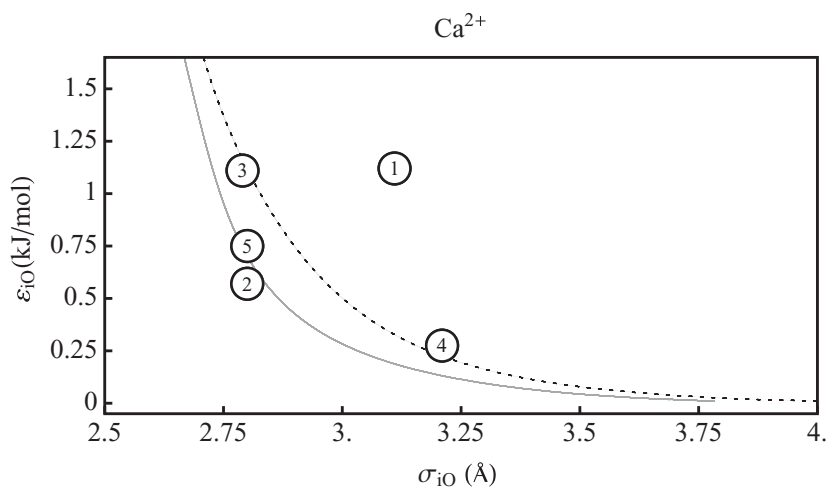

(c)

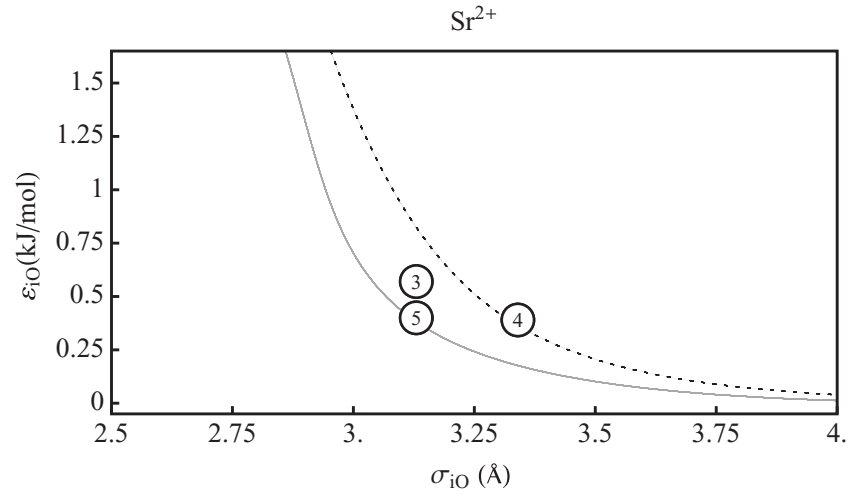

(b)

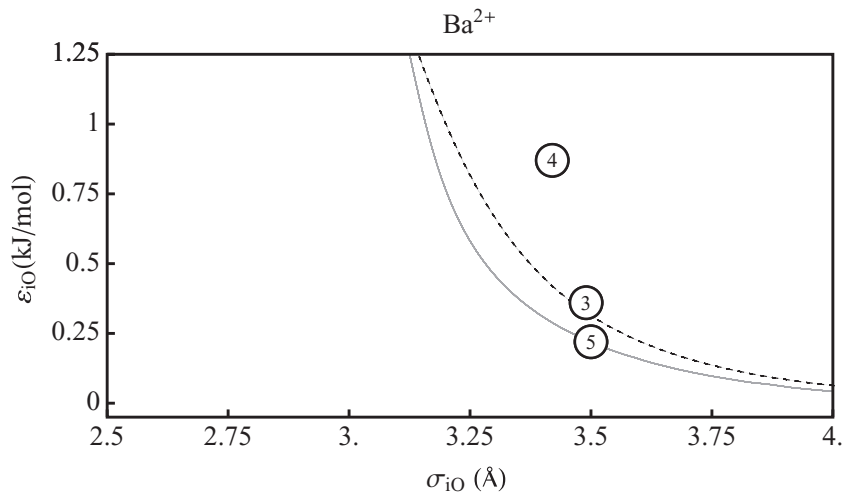

(d)

FIG. 4. LJ parameter plane for all divalent cations: $\mathrm{Mg}^{2+}, \mathrm{Ca}^{2+}, \mathrm{Sr}^{2+}, \mathrm{Ba}^{2+}$. Solid lines correspond to the combination of $\sigma_{i O}$ and $\varepsilon_{i O}$ that reproduce the sum of the experimental solvation free energies of the divalent ions and the reference chloride ion $\Sigma_{\Delta G}$. The dotted lines show the combinations that reproduce the experimental effective radius $R_{1}$. The open circles show the LJ parameters presented in Table I. Labels 1, 2, 3, 4, and 5 correspond to the AMBER FF, ${ }^{20}$ the Roux and Beglov ${ }^{17}$ or the CHARMM FF, the Åqvist FF, ${ }^{18}$ the Babu and Lim FF, ${ }^{19}$ and this work, respectively.

accurately describe $\mathrm{R}_{1}$, while others fail to reproduce any of these two.

Figure 5 compares the effective radius $\mathrm{R}_{1}$, calculated on the experimental iso-free-energetic solution line, as a function of $\sigma_{i O}$ with the experimental radius $\mathrm{R}_{1}$, denoted by horizontal lines, for the four cations. It is obvious that the experimental values are not exactly reproduced for any parameter combina-

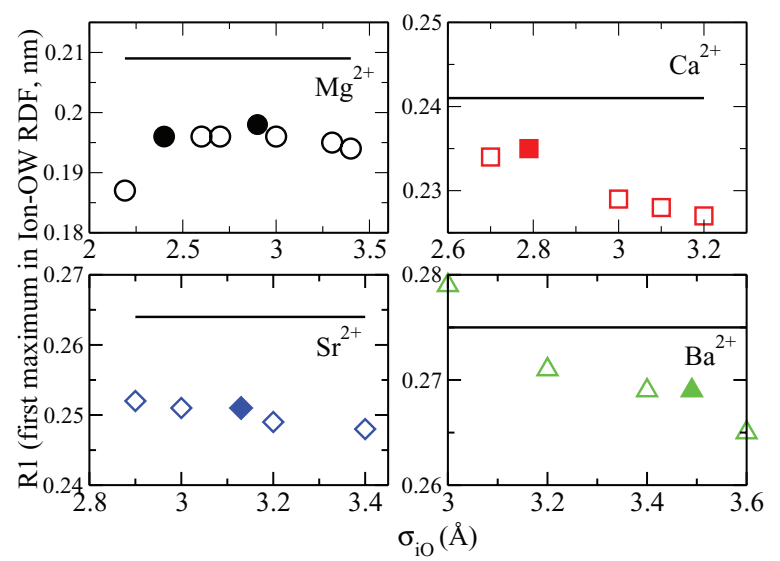

FIG. 5. Position of the first maximum in the cation-water radial distribution function $\mathrm{g}_{i O}$ (symbols) as a function of $\sigma_{i O}$ on the experimental solvation free energy isoline, compared with the experimental values (black horizontal lines). The filled symbols denote the optimized LJ force field parameters. Note that in the case of $\mathrm{Mg}^{2+}$ we have two optimal force fields. The concentration is $0.5 \mathrm{~m}$. tion. This is in line with the result shown in Fig. 4, revealing that the isolines for $\Sigma_{\Delta G}$ and $\mathrm{R}_{1}$ do not cross. As a consequence, the $\sigma_{i O}, \varepsilon_{i O}$ combinations that reproduce the free energies of solvation cannot simultaneously capture the cationwater effective radii. This discrepancy is a generic property of the Lennard-Jones 6-12 potential and not caused by any additional restriction or assumption made by us. Although this discrepancy is somewhat surprising, we point out that there is no a priori reason why it should be possible to simultaneously match the effective ion radius and the solvation free energies with a Lennard-Jones 6-12 potential. As we strive to optimize ionic force fields based on solvation and ion-pairing properties in the present study, this short-coming of the LennardJones 6-12 potential is not of main concern, we simply note in passing that it is conceivable that other potential forms perform better in this respect. However, as will be shown below, we will for the problematic case of $\mathrm{Mg}^{2+}$, where the activity derivative does not reach the experimental value, use the effective radius $R_{1}$ as a guideline to choose force fields to be used for further combination rule optimization.

\section{B. Activity coefficients}

The realistic balance between ion-ion and ion-water interactions is expressed by the experimental excess coordination numbers of ions in water $G_{c c}$ and $G_{c w}$ defined in Eqs. (14) and (15), which are related to the activity derivatives of 


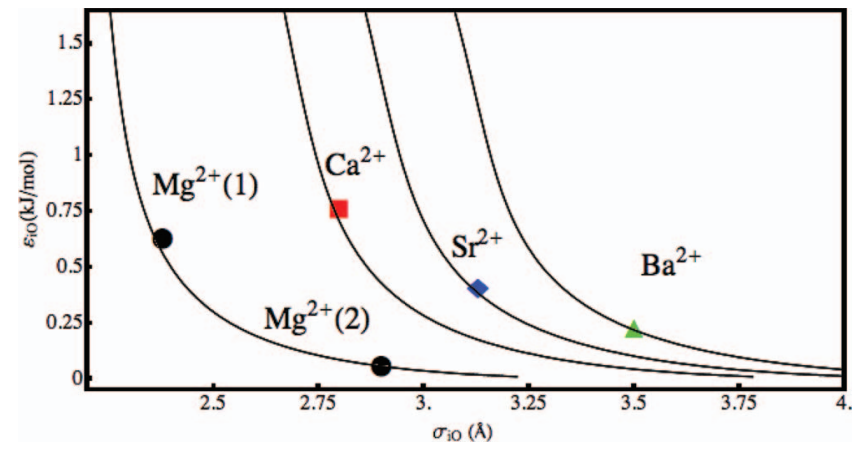

FIG. 6. The final $\mathrm{LJ}$ parameters for divalent cations: $\mathrm{Mg}^{2+}, \mathrm{Ca}^{2+}, \mathrm{Sr}^{2+}$, $\mathrm{Ba}^{2+}$. Solid lines correspond to the combinations of $\sigma_{i O}$ and $\varepsilon_{i O}$ that reproduce the sum of the experimental solvation free energies of the divalent ions and the reference chloride ion $\Sigma_{\Delta G}$. The symbols represent our optimized force field parameters, set (5) in Table I.

electrolytes. $^{24,25,47}$ Here, $G_{c c}$ measures the integrated excess of ions around other ions and $G_{c w}$ measures the integrated excess of water around ions. In the following, we choose combinations of LJ parameters that lie on the experimental free energy of solvation lines in Figs. 4 and 6 for all four divalent cations considered. For these LJ parameter combinations and for different divalent salt solutions we determine the KB integrals and compute the activity derivatives.

Using the Smith-Dang LJ parameter for the chloride ion, ${ }^{43}$ we first show the activity derivatives of $\mathrm{MgCl}_{2}, \mathrm{CaCl}_{2}$, $\mathrm{SrCl}_{2}$, and $\mathrm{BaCl}_{2}$ as a function of the LJ radius $\sigma_{i O}$ in Fig. 7. The simulation data follow more or less an inverse parabolic shape for all four cations. The experimental data for the activities as a function of salt molality are obtained from Ref. 49 and the corresponding activity derivatives are extracted using Eq. (13) and shown as horizontal colored lines. The activity derivative for $\mathrm{CaCl}_{2}$ with $\sigma_{i O}=2.8 \AA$ agrees with the experimental value within the numerical error and is smaller than the experimental value both for smaller and larger $\mathrm{LJ}$ radii. The activity derivative for $\mathrm{BaCl}_{2}$ matches the experimental data for $\sigma_{i O}=3.49 \AA$, while for $\mathrm{SrCl}_{2}$ we get good agreement for

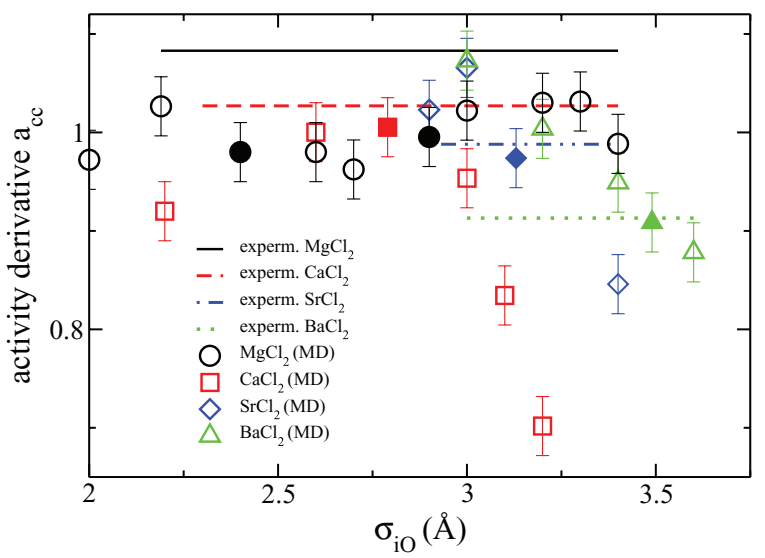

FIG. 7. Activity derivatives for $\mathrm{MgCl}_{2}, \mathrm{CaCl}_{2}, \mathrm{SrCl}_{2}$, and $\mathrm{BaCl}_{2}$ as a function of the ion-water $\mathrm{LJ}$ diameter $\sigma_{i O}$ of the divalent cations. The simulations are performed in the cubic box with $\mathrm{L}=40 \AA$ at $0.5 \mathrm{~m}$ electrolyte concentration. The symbols correspond to the simulated values, while the horizontal lines denote the respective experimental results at the same concentration. The filled symbols denote the optimized parameters. $\sigma_{i O}=3.13 \AA$. The optimal force field parameters are denoted by filled symbols in Figs. 6 and 7 .

The behavior of $\mathrm{MgCl}_{2}$ is distinctly different and deserves separate discussion. The simulated values of the activity derivative for $\mathrm{MgCl}_{2}$ lie between $\mathrm{a}_{c c}=0.96-1.02$ and do not reach the experimental value $\mathrm{a}_{c c}=1.09$ but rather show a broad plateau with a slight decrease at intermediate radii. For $\mathrm{Mg}^{2+}$ ions therefore the standard combination rule shown in Eq. (2) is not able to describe both solvation and activity properties simultaneously, which further below will be rectified by introducing a scaling factor for the anion-cation LJ radius. Because of the ambiguity of the simulated $\mathrm{a}_{c c}$ data as a function of the radius $\sigma_{i O}$, we choose two different $\mathrm{LJ}$ parameters for $\mathrm{Mg}^{2+}$ for this further optimization. First, we select a combination of $\sigma_{i O}$ and $\varepsilon_{i O}$ that reproduces the experimental solvation free energy exactly and $R_{1}$ with minimal error, as shown in Fig. 5. Our first parameter choice is $\sigma_{i O}=2.9 \AA$ and $\varepsilon_{i O}=0.051 \mathrm{~kJ} / \mathrm{mol}$. Our second choice is $\varepsilon_{i O}=0.62 \mathrm{~kJ} / \mathrm{mol}$ and $\sigma_{i O}=2.4 \AA$, which is similar to the $\sigma_{i O}$ value of the Åqvist force field and seems to correlate better with the radii obtained for the other, larger cations, as can be seen in Fig. 6 .

Before we describe the optimization of the combination rules, we briefly have a look at the Kirkwood-Buff factors in order to understand the quite characteristic behavior of the activity derivatives displayed in Fig. 7. Figure 8 shows running integrals of the $\mathrm{KB}$ integrals for $\mathrm{CaCl}_{2}$ at $0.5 \mathrm{~m}$ at different values of $\sigma_{i O}$ on the solvation free energy isoline. The running integrals saturate at distances beyond $10 \AA$, the oscillations in the $\mathrm{KB}$ integrals involving water attest to the pronounced structuring of the solvation shells. The saturated values of the $\mathrm{KB}$ integral values for $\mathrm{G}_{++}, \mathrm{G}_{+-}$, and $\mathrm{G}_{--}$first decrease with increasing $\mathrm{LJ}$ radius until $2.6 \AA$ and then increase at larger $\sigma_{i O}$ 's. This reflects the non-monotonic inverse-parabolic behavior of the activity coefficient derivative as a function of the $\mathrm{LJ}$ radius, which is the generic behavior for all cations shown in Fig. 7. If we look at the cation-anion and cation-oxygen

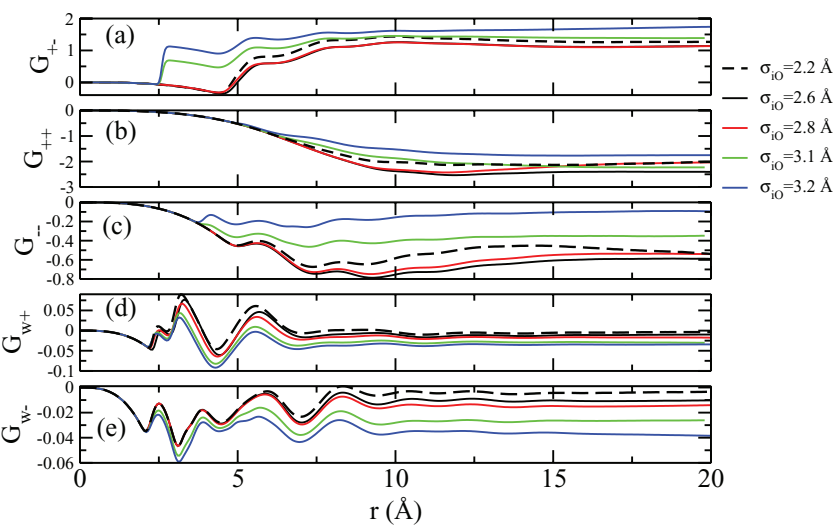

FIG. 8. Kirkwood-Buff running integrals as a function of the integration distance $\mathrm{r}$ for a $0.5 \mathrm{~m} \mathrm{CaCl}_{2}$ solution at different values of the $\mathrm{LJ}$ radius of $\sigma_{i O}$ on the experimental solvation free energy line. $\mathrm{G}_{+-}, \mathrm{G}_{++}, \mathrm{G}_{--}, \mathrm{G}_{w+}$, and $\mathrm{G}_{w-}$ represent Kirkwood-Buff integrals of cation-anion, cation-cation, anion-anion, water-cation, and water-anion pairs, respectively. Cation-anion Kirkwood-Buff integrals first decrease with increasing $\sigma_{i O}$, then increase with increasing of the $\mathrm{LJ}$ radius. Water-ion $\mathrm{KB}$ integrals are monotonically decreasing with increasing of $\sigma_{i O}$. 

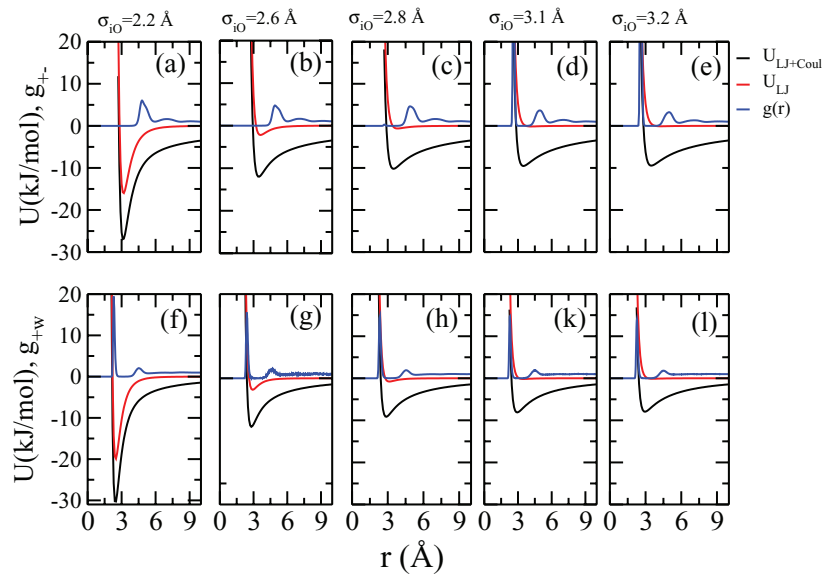

FIG. 9. Cation-anion (a, b, c, d, e) and cation-oxygen (f, g, h, k, l) radial distribution functions $g(r)$ for a $0.5 \mathrm{~m} \mathrm{CaCl}_{2}$ solution (blue lines), compared with the LJ potential, $\mathrm{U}_{L J}$ (red lines), and the sum of the $\mathrm{LJ}$ and Coulomb potentials, $\mathrm{U}_{L J}+\mathrm{U}_{\text {Coul }}$ (black lines), for various $\sigma_{i O}$ and $\varepsilon_{i O}$ parameters.

RDF's in Fig. 9, we see that at small radius $\sigma_{i O}=2.2 \AA$, Figs. 9(a) and 9(f), the $\mathrm{Ca}^{2+}$ ion is separated from the $\mathrm{Cl}^{-}$ ions by a strongly bound hydration shell. ${ }^{48}$ Nevertheless, the solvent-separated $\mathrm{Ca}^{2+}{ }_{-} \mathrm{Cl}^{-}$ion pair is energetically stabilized by the strong and favorable LJ interaction, which is shown in the same graph by red lines, while the black lines show the sum of the Coulomb and LJ interaction potentials (note that small LJ radius is equivalent to large $\mathrm{LJ}$ interaction strength on the experimental solvent-free energy line, see Fig. 4). For intermediate values of the LJ radius $\sigma_{i O}=2.6 \AA$ and $2.8 \AA$, Figs. 9(b) and 9(c), and 9(g) and 9(h), the solventseparated ion pair is energetically less favored because of the smaller LJ interaction strength, and the corresponding peak slightly diminishes. This leads to the increase of the activity derivative for intermediate radii. At large radii $\sigma_{i O}=3.1$ and $3.2 \AA$, Figs. 9(d) and $9(\mathrm{e})$, and $9(\mathrm{k})$ and $9(\mathrm{l})$, the small $\mathrm{LJ}$ interaction strength permits to form a direct $\mathrm{Ca}^{2+}-\mathrm{Cl}^{-}$ ion pair, giving rise to a strong peak in the RDF, a correspondingly large cation-anion $\mathrm{KB}$ integral $\mathrm{G}_{+-}$as seen in Fig. 8(a) and therefore a decreasing activity derivative, as seen in Fig. 7. This at first sight counter-intuitive shift from solvent-separated ion pairing for small $\sigma_{i O}$ to direct ion pairing for large $\sigma_{i O}$ is at the core of the inverse parabolic behavior of $a_{c c}$ seen in Fig. 7.

\section{Modified mixing rules and transferability}

It is clear from the data shown in Fig. 7 that LJ parameter combinations that reproduce the $\mathrm{Mg}^{2+}$ solvation free energy fail to reproduce the experimental activity coefficient derivatives of $\mathrm{MgCl}_{2}$ solutions. To overcome this problem we follow a recently proposed scheme, which modifies the Lorentz-Berthelot combination rule for the cation-anion effective radius and was used to reproduce the experimental activity derivatives of monovalent salt solutions involving the problematic halide ions $\mathrm{F}^{-}$and $\mathrm{I}^{-} .{ }^{24}$ In that work, a freely adjustable scaling factor $\lambda_{\sigma}$ was introduced in the cation-anion effective radius

$$
\sigma_{+-}=\lambda_{\sigma}\left(\sigma_{+}+\sigma_{-}\right) / 2
$$

and varied such as to reproduce the experimental activity derivative. The LJ parameters we use for the divalent cations lie on the free energy solvation curves in Fig. 6 and are denoted by filled symbols in Fig. 7. These cationic force fields carry the label " 5 " in Table I. We extend our analysis also to bromide and iodide salts and thereby test the transferability of our force fields for divalent cations. The LJ pair parameters for the monovalent anions of $\mathrm{Cl}, \mathrm{Br}$, and I are listed in Table II and are taken from Refs. 35 and 43. We repeat that we keep the ion-water LJ parameters fixed during this last step of optimization, which is based on a combination of single-ion solvation free energy and ion-pair activity derivative matching, as explained earlier, and only modify the cation-anion effective $\mathrm{LJ}$ radius. The cation-cation, anion-anion, and waterion mixing rules are not modified. This reflects the expectation that the effective water-ion radii are not expected to change due to the presence of other ions and stands in contrast to other studies. ${ }^{25,44}$

The cation-anion scaling prefactor $\lambda_{\sigma}$ is varied in discrete steps of $\lambda_{\sigma}=0.8,1.0,1.2,1.5,1.6,1.7,2.0$. In Fig. 10 we summarize the results for the activity derivatives $\mathrm{a}_{c c}$ as a function of the scaling prefactor $\lambda_{\sigma}$ at $0.5 \mathrm{~m}$ concentration separately for all four divalent cations and for the three halide ions considered. The experimental values for $\mathrm{a}_{c c}$ are denoted by horizontal lines. ${ }^{49}$ The symbols show the simulated activity derivatives and solid lines denote cubic spline fits. Note that for $\mathrm{Mg}^{2+}$ in Fig. 10(a) we display results for two different force fields. A satisfactory match between simulated and experimental activity derivatives for $\mathrm{MgCl}_{2}$ (black) and $\mathrm{MgBr}_{2}$ (red) is obtained around $\lambda_{\sigma}=1.6$ for both force fields. For $\mathrm{MgI}_{2}$ (green), both force fields slightly differ, and a good match between simulation and experimental data is obtained for force field (1) for $\lambda_{\sigma}=1.5$ and for force field (2) for $\lambda_{\sigma}=1.7$. All fitting results for the scaling prefactor are summarized in Table III.

As already seen in Fig. 7 where we showed the activity derivative for the unmodified mixing rule, the situation for $\mathrm{Ca}^{2+}$ is more favorable. For $\mathrm{CaCl}_{2}$ (black) and $\mathrm{CaBr}_{2}$ (red) the activity derivatives match the experimental data without the need for modifying the mixing rule, as seen in Fig. 10(b). For these salts the unmodified factor $\lambda_{\sigma}=1.0$ is just doing fine. For $\mathrm{CaI}_{2}$ a slightly decreased scaling prefactor of $\lambda=0.8$ similar to our previous study for monovalent cations ${ }^{24}$ gives perfect agreement with experiments, but we note that the error

TABLE III. Optimal scaling prefactors $\lambda_{\sigma}$ for the cation-anion LJ effective radius, obtained from the interpolated intersection between the experimental activity derivative and the fitted simulation curves in Fig. 10. The last column lists the suggested transferable scaling prefactor $\bar{\lambda}_{\sigma}$ which approximately holds for all anions.

\begin{tabular}{lcccc}
\hline \hline & \multicolumn{4}{c}{$\lambda_{\sigma}$} \\
\cline { 2 - 4 } Ion & $\mathrm{Cl}^{-}$ & $\mathrm{Br}^{-}$ & $\mathrm{I}^{-}$ & $\bar{\lambda}_{\sigma}$ \\
\hline $\mathrm{Mg}^{2+}(1)$ & 1.6 & 1.6 & 1.5 & 1.6 \\
$\mathrm{Mg}^{2+}(2)$ & 1.6 & 1.6 & 1.7 & 1.6 \\
$\mathrm{Ca}^{2+}$ & 1.0 & 1.0 & 0.8 & 1.0 \\
$\mathrm{Sr}^{2+}$ & 1.0 & 1.0 & 0.9 & 1.0 \\
$\mathrm{Ba}^{2+}$ & 1.0 & 1.0 & 0.95 & 1.0 \\
\hline
\end{tabular}



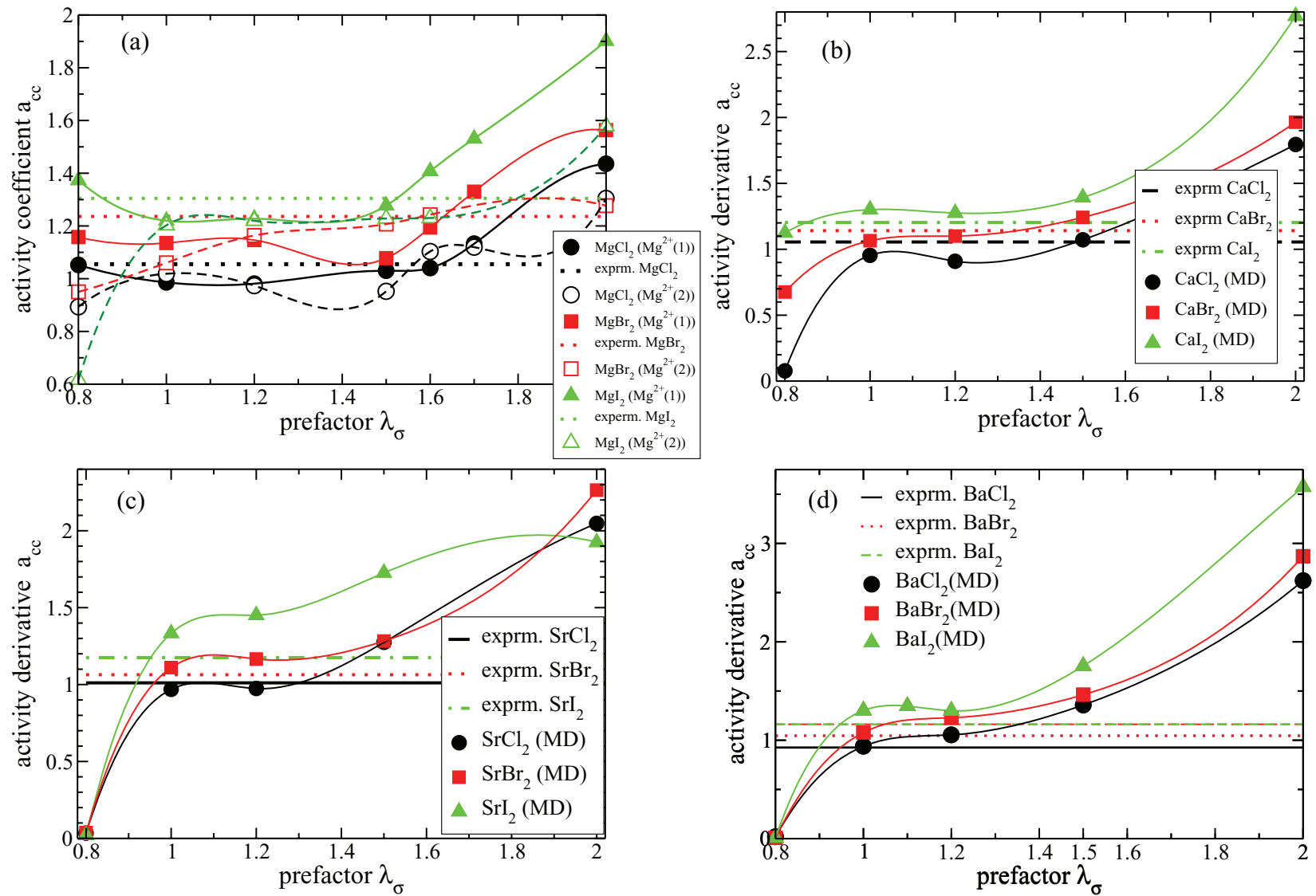

FIG. 10. The activity derivative as a function of the scaling prefactor $\lambda_{\sigma}$ at concentration $0.5 \mathrm{~m}$ for (a) $\mathrm{Mg}^{2+}$, (b) $\mathrm{Ca}^{2+}$, (c) $\mathrm{Sr}^{2+}$, and (d) $\mathrm{Ba}^{2+}$ salt solutions, respectively. For magnesium we show results for two different LJ parameter combinations of $\sigma_{i O}=2.4 \AA, \varepsilon_{i O}=0.62 \mathrm{~kJ} / \mathrm{mol}\left(\right.$ filled symbols, Mg$\left.{ }^{2+}(1)\right)$ and $\sigma_{i O}=2.9 \AA, \varepsilon_{i O}=0.05 \mathrm{~kJ} / \mathrm{mol}$ (open symbols, $\mathrm{Mg}^{2+}(2)$ ). The symbols show simulation results, curves are spline fitting functions. The horizontal lines denote the corresponding experimental activity derivative values.

one makes when choosing $\lambda_{\sigma}=1.0$ is minimal. Apparently, iodide in conjunction with a cation is less repulsive than when interacting with water, which is reflected by a smaller cationanion effective radius and therefore $\lambda_{\sigma}$ smaller than unity.

For the remaining salts $\mathrm{SrY}_{2}$ and $\mathrm{BaY}_{2}$ shown in Figs. $10(\mathrm{c})$ and $10(\mathrm{~d})$ the situation is straightforward. Here the unmodified combination rule with $\lambda_{\sigma}=1.0$ reproduces the experimental activity derivatives quite well. Only for the iodide salts is the simulated value of $\mathrm{a}_{c c}$ slightly higher than the experimental value and the optimal scaling prefactor is less than unity. In fact, for $\mathrm{SrI}_{2}$ in Fig. 10(c) we predict an optimal value of $\lambda_{\sigma}=0.9$ by interpolation, for $\mathrm{BaI}_{2}$ in Fig. 10(d) we predict an optimal value of $\lambda_{\sigma}=0.95$.

Altogether, the data in Fig. 10 demonstrate that the scaling prefactor $\lambda_{\sigma}$ of the cation-anion effective radius is a very efficient means to increase the salt activity derivative. Apart from a quasi-plateau of $\mathrm{a}_{c c}$ in the range $\lambda_{\sigma}=1.0-1.5$ observed for all salt solutions, the activity derivative steadily increases with growing $\lambda_{\sigma}$. The quasi-plateau can again be traced back to a shift from direct ion-pairing to solvent-separated ion pairing, similar to what was seen in Fig. 9. This is visualized in Fig. 11 where we show cation-anion RDF's for $\mathrm{CaCl}_{2}$ for several values of $\lambda_{\sigma}$. Only for $\lambda_{\sigma}=0.8$ (black line) is the direct ion pair prominent, for all higher values of $\lambda_{\sigma}$ the solventseparated ion pair is statistically more significant. We see that the optimal force fields for divalent cations are close to the transition between direct and solvent-separated ion pairing, which explains why robust optimization strategies are needed in order to bring out the subtle differences between the various cations considered in this study.

Table III lists the final scaling factors obtained through the comparison of the simulation data to the experimental activity derivatives in Fig. 10 for all ion combinations used in

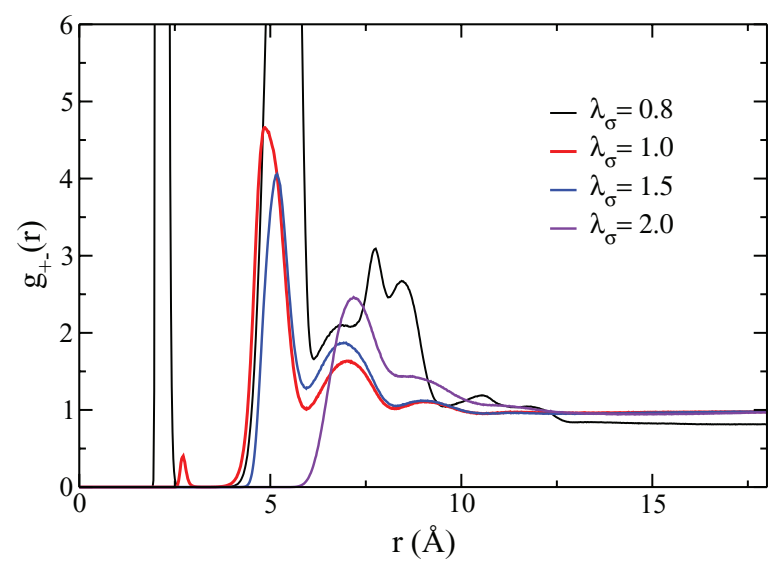

FIG. 11. Radial cation-anion distribution function $g_{+-}$for a $0.5 \mathrm{~m} \mathrm{CaCl}_{2}$ solution for different values of the scaling prefactor $\lambda_{\sigma}$. With increasing $\lambda_{\sigma}$ the direct ion pair peak of the RDF decreases and the whole distribution shifts to larger cation-anion distances, which explains the increase of $\mathrm{a}_{c c}$ as $\lambda_{\sigma}$ grows. 
this study. For $\mathrm{Sr}^{2+}$ and $\mathrm{Ba}^{2+}$ the unmodified mixing rules work fine and a scaling factor of $\lambda_{\sigma} \simeq 1$ describes cation-anion quite well. For $\mathrm{Ca}^{2+}$ deviations are seen for $\mathrm{CaI}_{2}$, while for $\mathrm{Mg}^{2+}$ the scaling factors are significantly larger than unity for both $\mathrm{LJ}$ parameters we considered. The two $\mathrm{Mg}^{2+}$ force fields are of equal quality, as far as we can judge based on the data we have. Force field (1) with a smaller radius of $\sigma_{i O}$ $=2.4 \AA$ seems more consistent with the radii of the other divalent cation force fields in Fig. 6, but we note that this is a purely cosmetic argument.

When comparing scaling prefactors for one cation and different anions, one sees approximate transferability emerging, i.e., the difference of $\lambda_{\sigma}$ between the various anions are much smaller than the difference between $\mathrm{Ca}^{2+}$ and $\mathrm{Mg}^{2+}$. To reflect this, we define a mean scaling factor $\bar{\lambda}_{\sigma}$ for each cation that can be uniformly used for all anions and which represents the approximate transferability of the divalent cation force field.

\section{CONCLUSIONS}

In the present study divalent cation force field parameters are developed in conjunction with the SPC/E water model through molecular dynamics simulations. The parameters are derived on the basis of experimental solvation free energies and activity derivatives of salt solutions. Absolute solvation free energies of single ions are ambiguous because they are strongly affected by the air-water interface potential. Therefore we use the solvation free energies of neutral ion-pairs. As an additional check on the quality of the derived force fields we also determine the effective radii of ions from the first peak of the radial ion-oxygen water distribution function. Activity derivatives are obtained through Kirkwood-Buff solution theory. For some of the cations studied, namely $\mathrm{Ca}^{2+}, \mathrm{Sr}^{2+}$ and $\mathrm{Ba}^{2+}$, the simultaneous optimization of solvation free energies and activity derivatives is possible with the standard combination rules (with small deviations for the iodide salts). For $\mathrm{Mg}^{2+}$, on the other hand, this double optimization is not possible. This reflects the known problem of resolving the experimentally known differences between $\mathrm{Ca}^{2+}$ and $\mathrm{Mg}^{2+}$ ions in classical MD simulations, which is a pressing issue as these ions are physiologically very important. As a solution to this issue, we modify the combination rule for the effective cation-anion radius, following previous work on monovalent cations. ${ }^{24}$ It turns out that the effective radius of the $\mathrm{Mg}^{2+}$ ion with respect to the anion has to be increased by a factor of $\lambda_{\sigma}=1.6$, which shows that the correction is not minor. When extending the analysis to the activity derivatives for the salt solutions $\mathrm{MgY}_{2}, \mathrm{CaY}_{2}, \mathrm{SrY}_{2}, \mathrm{BaY}_{2}$, with $\mathrm{Y}=\mathrm{Cl}, \mathrm{Br}$, I, we find approximate transferability to hold, i.e., the scaling factor $\lambda_{\sigma}$ is approximately constant for the different anions considered.

We note that the ordering of the experimental activity coefficients ${ }^{49,50}$ according to $\mathrm{Mg}^{+2}>\mathrm{Ca}^{+2}>\mathrm{Sr}^{+2}>\mathrm{Ba}^{+2}$ implies that the smaller $\mathrm{Mg}^{+2}$ ion binds less strongly to the halide ions than the larger cations. Such an ordering of the activity coefficients is well-known from other ions as well and expressed in terms of the so-called Hofmeister series, ${ }^{51}$
TABLE IV. Comparison of experimental and simulated diffusion coefficients for different salt solutions involving divalent cations at a concentration of $0.5 \mathrm{~m}$. The force fields parameters are the optimized ones according to Tables I and III. Labels (1) and (2) for $\mathrm{Mg}^{+2}$ imply the first and second model for $\mathrm{Mg}^{+2}$ according to Table I. See Fig. 1 in the supplementary material ${ }^{55}$ for the center-of-mass mean square displacement leading to the diffusion coefficients.

\begin{tabular}{lcc}
\hline \hline Ion & $\mathrm{D}(\operatorname{sim}) \times 10^{-5} \mathrm{~cm}^{2} / \mathrm{s}$ & $\mathrm{D}(\exp ) \times 10^{-5} \mathrm{~cm}^{2} / \mathrm{s}($ Ref. 54) \\
\hline $\mathrm{Mg}^{2+}(1)$ & $1.13 \pm 0.01$ & 1.0635 \\
$\mathrm{Mg}^{2+}(2)$ & $1.10 \pm 0.01$ & 1.0635 \\
$\mathrm{Ca}^{2+}$ & $1.08 \pm 0.01$ & 1.14 \\
$\mathrm{Sr}^{2+}$ & $0.92 \pm 0.01$ & 1.20 \\
$\mathrm{Ba}^{2+}$ & $0.80 \pm 0.01$ & 1.16 \\
\hline \hline
\end{tabular}

but we note that the Hofmeister series for one set of ions is frequently inverted depending on the counter ion or the specific situation considered. ${ }^{52}$ As a matter of fact, the experimental ordering of the activity coefficients of the divalent cations, and consequently also the ordering of our forcefields which are constructed to reproduce the experimental results, seem to contradict the Lewis-acid concept for divalent cations: According to this concept $\mathrm{Mg}^{+2}$ is a stronger Lewis acid than $\mathrm{Ca}^{+2}$ and should therefore, due to its small size, binds stronger to a negative charge. ${ }^{53}$ The contradiction is only an apparent one and resolved by the observation that ionpairs involving divalent cations are predominantly solventseparated, meaning that $\mathrm{Mg}^{+2}$ bind more strongly its water hydration water shell and therefore binds less strongly to the halide anions, which is reflected by the large anion-cation radius factor $\lambda_{\sigma}$ of $\mathrm{Mg}^{+2}$.

Another notorious issue related to transferability is whether the force fields we have developed based on static ion-pair properties are also able to describe dynamic quantities. As a representative dynamic quantity we calculate the diffusion coefficient of different salt solutions and compare with the respective experimental data, ${ }^{54}$ the results are shown in Table IV for a concentration of $0.5 \mathrm{~m}$ (more details on the center-of-mass mean square displacement can be found in Fig. 1 in the supplementary material). ${ }^{55}$ The agreement between simulations and experiments for $\mathrm{MgCl}_{2}$ and $\mathrm{CaCl}_{2}$ is acceptable, but for $\mathrm{SrCl}_{2}$ and $\mathrm{BaCl}_{2}$ a sizable discrepancy is found. In fact, the experimental trend, showing that larger cations have a larger diffusion coefficient, is missed in the simulations. This shows that the optimization procedure we have used, based on static properties, does not simultaneously reproduce kinetic properties of salt solutions involving divalent cations. More work along these lines seems required.

As a final remark, the LJ parameters for the divalent cations proposed here were optimized at $0.5 \mathrm{~m}$ and therefore are expected to be accurate in the dilute and intermediate concentration regime. For higher concentrations, aggregation and clustering effects can in principle occur. As another comment, the transferability of the proposed cationic force fields has been checked for $\mathrm{Cl}^{-}, \mathrm{Br}^{-}$, and $\mathrm{I}^{-}$, but remains to be scrutinized for other, biologically relevant anionic groups. 


\section{ACKNOWLEDGMENTS}

Sh.M. acknowledges financial support from the Marie Curie International Incoming Fellowship (call FP7-PEOPLE2009-IIF). M.F. acknowledges support from the "BioFuS" project of the Intra-European Marie-Curie Programm (call FP7-PEOPLE-2009-IEF) and the Deutsche Forschungsgemeinschaft (DFG) as part of the SFB 716 "Dynamische Simulation von Systemen mit großen Teilchenzahlen". R.R.N. acknowledges support from the DFG as part of the SFB 1078 "Protonation Dynamics in Protein Function." Fruitful discussions with J. Smiatek are also acknowledged.

${ }^{1}$ D. A. Doyle, J. M. Cabral et al., Science 280, 69 (1998).

${ }^{2}$ Y. X. Jiang, A. Lee, J. Y. Chen, V. Ruta, M. Cadene, B. T. Chait, and R. MacKinnon, Nature (London) 423, 33 (2003).

${ }^{3}$ R. Dutzler, E. B. Campbell, M. Cadene, B. T. Chait, and R. MacKinnon, Nature (London) 415, 287 (2002).

${ }^{4}$ A. Malovikova, M. Rinaudo, and M. Milas, Biopolymers 34, 1059 (1994).

${ }^{5}$ F. G. Prendergast and K. Mann, J. Biol. Chem. 252, 840 (1977).

${ }^{6}$ S. X. Wang, E. Hur, C. A. Sousa, L. Brinen, E. J. Slivka, and R. J. Fletterick, Biochemistry 42, 7959 (2003).

${ }^{7}$ G. Kartha, K. I. Varughese, and S. Aimoto, Proc. Natl. Acad. Sci. U.S.A. 79, 4519-4522 (1982).

${ }^{8}$ J. Marra and J. Israelachvili, Biochemistry 24, 4608-4618 (1985).

${ }^{9}$ J. Wilschut, M. Holsappel, and R. Jansen, Biochim. Biophys. Acta 690, 297-301 (1982).

${ }^{10}$ T. Dudev and L. Carmay, Annu. Rev. Biophys. 37, 97 (2008).

${ }^{11}$ T. Dudev and L. Carmay, Chem. Rev. 103, 773 (2003).

${ }^{12}$ T. A. Thomas, A. Halgren, and W. Damm, Curr. Opin. Struct. Biol. 11, 236-242 (2001).

${ }^{13}$ W. L. Ash, M. R. Zlomislic, E. O. Oloo, and D. P. Tieleman, Biochim. Biophys. Acta 1666, 158-189 (2004).

${ }^{14}$ P. G. Kusalik and G. N. Patey, J. Chem. Phys. 86, 5110 (1987).

${ }^{15}$ F. P. Kirkwood and J. G. Buff, J. Chem. Phys. 19, 774 (1951).

${ }^{16}$ A. P. Lyubartsev and A. Laaksonen, Phys. Rev. E 55, 5689 (1997).

${ }^{17}$ D. Beglov and B. Roux, J. Chem. Phys. 100, 9050-9063 (1994).

${ }^{18}$ J. Åqvist, J. Phys. Chem. 94, 8021 (1990).

${ }^{19}$ C. S. Babu and C. Lim, J. Phys. Chem. 110, 691-699 (2006).

${ }^{20}$ D. A. Pearlman, D. A. Case, J. W. Caldwell, W. S. Ross, T. E. Cheatham, S. DeBolt, D. Ferguson, G. Seibel, and P. Kollman, Comput. Phys. Commun. 91, 1-41 (1995).

${ }^{21}$ T. Bastug and S. Kuyucak, Chem. Phys. Lett. 424, 82-85 (2006).

${ }^{22}$ T. Bastug and S. Kuyucak, Biophys. J. 90, 3941-3950 (2006).

${ }^{23}$ M. Patra and M. Karttunen, J. Comput. Chem. 25, 678 (2004).

${ }^{24}$ M. Fyta and R. R. Netz, J. Chem. Phys. 136, 124103 (2012).
${ }^{25}$ M. B. Gee et al., J. Chem. Theory Comput. 7, 1369 (2011).

${ }^{26}$ B. R. Brooks, R. E. Bruccoleri, B. D. Olafson, D. J. States, S. Swaminathan, and M. Karplus, J. Comput. Chem. 4, 187 (1983).

${ }^{27}$ J. R. Grigera, H. J. C. Berendsen, and T. P. Straatsma, J. Phys. Chem. 91, 6269 (1987).

${ }^{28}$ J. P. Ryckaert, G. Ciccotti, and H. J. C. Berendsen, J. Comput. Phys. 23, 327-341 (1977).

${ }^{29}$ T. P. Straatsma and J. A. Mccammon, Ann. Rev. Phys. Chem. 43, 407-435 (1992).

${ }^{30}$ H. J. C. Berendsen, D. van der Spoel, and R. van Drunen, Comput. Phys. Commun. 91, 43 (1995).

${ }^{31}$ E. Lindahl, B. Hess, and D. van der Spoel, J. Mol. Model. 7, 306 (2001).

${ }^{32}$ T. Darden, D. York, and L. Pedersen, J. Chem. Phys. 98, 10089-10092 (1993).

${ }^{33}$ H. J. C. Berendsen, J. P. M. Postma, W. F. van Gunsteren, A. DiNola, and J. R. Haak, J. Chem. Phys. 81, 3684 (1984).

${ }^{34}$ M. Fyta, I. Kalcher, L. Vrbka, J. Dzubiella, and R. R. Netz, J. Chem. Phys. 132, 024911 (2010).

${ }^{35}$ D. Horinek, S. I. Mamatkulov, and R. R. Netz, J. Chem. Phys. 130, 124507 (2009).

${ }^{36}$ G. Hummer, L. Pratt, A. Garcia, B. Berne, and S. Rick, J. Phys. Chem. B 101, 3017 (1997).

${ }^{37}$ M. Rami Reddy and M. L. Berkowitz, Chem. Phys. Lett. 155, 173 (1989).

${ }^{38}$ T. Darden, D. Pearlman, and L. G. Pedersen, J. Chem. Phys. 109, 10921 (1998).

${ }^{39}$ G. Hummer, L. Pratt, and A. Garcia, J. Phys. Chem. 100, 1206 (1996).

${ }^{40}$ G. Lee Warren and S. Patel, J. Chem. Phys. 127, 064509 (2007).

${ }^{41}$ M. D. Tissandier, K. A. Cowen et al., J. Chem. Phys. 102, 7787-7794 (1998).

${ }^{42}$ Y. Marcus, Ion Properties (Marcel Dekker, New York, Basel, 1997).

${ }^{43}$ L. X. Dang and D. E. Smith, J. Chem. Phys. 99, 6950-6956 (1993).

${ }^{44}$ S. Weerasinghe and P. E. Smith, J. Chem. Phys. 119, 11342 (2003).

${ }^{45}$ A. Ben-Naim, Statistical Thermodynamics for Chemists and Biochemists (Plenum, New York, 1992).

${ }^{46}$ A. P. Lyubartsev and S. Marcelja, Phys. Rev. E 65, 041202 (2002).

${ }^{47}$ B. Hess and N. A. F. van der Vegt, Proc. Natl. Acad. Sci. U.S.A. 106, 13296-13300 (2009).

${ }^{48}$ K. D. Collins, Biophys. J. 72, 65-76 (1997).

${ }^{49}$ R. A. Robinson and R. H. Stokes, Electrolyte Solutions, 2nd ed. (Dover, New York, 2002).

${ }^{50}$ J. P. Simonin, S. Krebs, and W. Kunz, Ind. Eng. Chem. Res. 45, 4345-4354 (2006).

${ }^{51}$ W. Kunz, Specific Ion Effects, 1st ed. (World Scientific, Singapore, 2010).

${ }^{52}$ N. Schwierz and R. R. Netz, Langmuir 28, 3881-3886 (2012).

${ }^{53}$ R. G. Pearson, J. Am. Chem. Soc 107, 6801-6806 (1985).

${ }^{54}$ V. M. Lobo, Pure Appl. Chem. 65, 2613-2640 (1993).

${ }^{55}$ See supplementary material at http://dx.doi.org/10.1063/1.4772808 for Table of solvation free energies, corresponding correction terms and mean square displacement of ions. 